\title{
Fault Diagnosis of Planetary Gear Based on FRWT and 2D-CNN
}

\author{
Jie Ma ${ }^{1}{ }^{1}$ and Lei Jiao ${ }^{2}$ \\ ${ }^{1}$ School of Mechanical and Electrical Engineering, Beijing Information Science and Technology University, Beijing, China \\ ${ }^{2}$ School of Automation, Beijing University of Information Science and Technology, Beijing, China \\ Correspondence should be addressed to Jie Ma; mjbeijing@163.com
}

Received 23 August 2021; Accepted 6 January 2022; Published 10 February 2022

Academic Editor: Meng Li

Copyright $(2022$ Jie Ma and Lei Jiao. This is an open access article distributed under the Creative Commons Attribution License, which permits unrestricted use, distribution, and reproduction in any medium, provided the original work is properly cited.

The fault signals of planetary gears are nonstationary and nonlinear signals. It is difficult to extract weak fault features under strong background noise. This paper adopts a new filtering method, fractional Wavelet transform (FRWT). Compared with the traditional fractional Fourier transform (FRFT), it can improve the effect of noise reduction. This paper adopts a planetary gear fault diagnosis method combining fractional wavelet transform (FRWT) and two-dimensional convolutional neural network (2DCNN). Firstly, several intrinsic mode component functions (IMFs) are obtained from the original vibration signal by AFSA-VMD decomposition, and the two components with the largest correlation coefficient are selected for signal reconstruction. Then, the reconstructed signal is filtered in fractional wavelet domain. By analyzing the wavelet energy entropy of the filtered signal, a twodimensional normalized energy characteristic matrix is constructed and the two-dimensional features are input into the twodimensional convolution neural network model for training. The simulation results show that the training effect of this method is better than that of FRFT-2D-CNN. Through the verification of the test set, we can know that the fault diagnosis of planetary gears can be realized accurately based on FRWT and 2D-CNN.

\section{Introduction}

As an important part of rotating machinery and equipment, planetary gears usually operate in a high-speed and highpower environment. They are widely used in aircraft manufacturing, coal mining machinery, wind power generation, ship manufacturing, and other industries. It is very easy to appear in the long-term operation process, smooth vibration phenomenon. Since the 1980s, many serious accidents have been caused by the fault of rotating equipment around the world, causing huge economic losses. About $80 \%$ of the faults occurred on the planetary gears [1]. Therefore, how to accurately diagnose the fault of planetary gears has important research significance.

At present, many achievements have been made in the research on fault diagnosis of planetary gears. Yu Jun and others proposed a planetary gear fault identification method that combines a stacked denoising autoencoder (SDAE) and a gated recurrent unit neural network (GRUNN) to solve the problem of low planetary gear fault recognition rate [1]. Gao Hongying and others proposed a planetary gear fault identification method combining complementary set empirical mode decomposition (CEEMD) and chaotic particle swarm kernel extreme learning machine (CPSO-ELM), which reduces the influence of external disturbances on planetary gear fault diagnosis [2]. Wang Zhenya and others proposed a fault diagnosis method based on optimized variational modal decomposition and multidomain manifold learning of the salvia group, which solved the problem of difficult feature extraction and identification of planetary gears [3]. Li Haiping proposed an intelligent diagnosis method combining Fast Fourier Transform (FFT) and Deep Confidence Network (DBN) to improve the accuracy of planetary gear fault diagnosis [4]. Li Yuheng proposed a fault diagnosis method that combines the ensemble empirical mode (EEMD) and the symmetrical differential energy operator to achieve accurate diagnosis of planetary gears and accurately obtain the fault characteristic frequency value of planetary gears [5]. Zhang et al. proposed a fault diagnosis method based on time-frequency characteristics and PSOSVM, and verified that the method can quickly and 
accurately identify the fault type of planetary gears from nonstationary signals [6]. Wang et al. proposed a gear fault diagnosis method based on multicriteria fault feature selection and heterogeneous integrated learning classification, which improved the accuracy and robustness of diagnosis [7]. Aiming at a kind of multimode process with hidden degenerate faults, a fault prediction algorithm based on the combination of multi-PCA model and fault reconstruction technology is proposed, which can well solve the fault prediction problem of multimode process data [8].

In order to realize the planetary gear fault diagnosis under strong background noise, this paper adopts the planetary gear fault diagnosis method combining fractional wavelet transform and two-dimensional convolutional neural network. Firstly, the planetary gear fault signal is denoised by fractional wavelet transform. Secondly, use wavelet packet to extract the one-dimensional normalized energy value of the filtered signal, and convert the obtained one-dimensional energy value into a two-dimensional energy feature map. Finally, use a two-dimensional convolutional neural network to establish a fault diagnosis model to achieve accurate identification of different faults under different working conditions.

\section{The Theoretical Basis of Fractional Wavelet Transform}

2.1. Discrete Wavelet Transform. In signal processing, the continuous wavelet is discretized. After the discretization, the continuous wavelet and its corresponding wavelet transform become the discrete wavelet transform. The discrete wavelet transform [8] is the second of the displacement and scale of the continuous wavelet transform. The power is discretized, which is essentially binary wavelet transform. In order to reduce the complexity of wavelet coefficients, the wavelet coefficients are taken at some discrete points, and the scale is discretized first. In order to reduce the wavelet transform coefficients of the remainder, we set the wavelet system. In order to reduce the wavelet transform coefficients of the remainder, we limit the values of $a$ and $b$ of the wavelet coefficient $\psi_{a, b}(t)=1 / \sqrt{a} \psi(t-b / a)$ to some discrete points and first discretize the scale, that is, let $a=a_{0}^{j} a_{0}>0$. At this time, the corresponding wavelet function is $a_{0}^{-j / 2} \psi\left[a_{0}^{-j}(t-b)\right]$, where $j=0,1,2, \ldots, b$ is a uniform discrete value. At scale $j$, since the width of $\psi\left(a_{0}^{-j} t\right)$ is $a_{0}^{j}$ times $\psi(t)$, the interval can be used to expand $a_{0}^{j}$ without changing $\psi(t)$. At this time, $\psi_{a, b}(t)$ is changed to

$$
\begin{aligned}
\psi_{a, b}(t) & =a_{0}^{-j / 2} \psi\left[a_{0}^{-j}\left(t-k a_{0}^{b} \tau b_{0}\right)\right] \\
& =a_{0}^{-j} \psi\left[a_{0}^{-j} t-k b_{0}\right] .
\end{aligned}
$$

The discrete wavelet transform is

$$
\begin{aligned}
W T\left(a_{0}^{-j} t-k b_{0}\right)=\int f(t) \psi_{a_{0}^{j}, k b_{0}}(t) \mathrm{d} t \\
\\
j=0,1,2, \ldots, k \in z .
\end{aligned}
$$

The continuous wavelet transform at $a=2^{j}(j \in z)$ is called discrete binary wavelet transform, and its expression is

$$
\begin{aligned}
\omega_{j}\left(2^{j}, b\right) & \leq f(t), \\
\psi_{2^{j}, b}(t) & \geq 2^{-1 / 2} \int f(t) \psi^{*}\left(\frac{t-b}{2^{j}}\right) \mathrm{d} t .
\end{aligned}
$$

2.2. Discrete Fractional Fourier Transform. The fractional Fourier transform is

$$
X_{p}(u)=\int_{-\infty}^{+\infty} K_{p}\left(u, u^{\prime}\right) x\left(u^{\prime}\right) \mathrm{d} u^{\prime} .
$$

$K_{P}\left(u, u^{\prime}\right)=A_{\alpha} \exp \left[j \pi\left(u^{2} \cot \alpha-2 u u^{\prime} \csc \alpha+u^{\prime 2} \cot \alpha\right)\right]$ is called the kernel function of FRFT, $A_{\alpha}=\sqrt{1-j \cot \alpha}$, $\alpha=p \pi / 2, p \neq 2 n, n$ is integers.

According to the definition given by formula (4), the formula of Ozaktas sampling fractional Fourier transform can be obtained as

$$
\begin{aligned}
X_{P}(u)= & A_{\alpha} \int_{-\infty}^{+\infty} \exp \left[j \pi\left(u^{2} \cot \alpha-2 u u^{\prime} \csc \alpha+{u^{\prime 2}}^{2} \cot \alpha\right)\right] \\
& x\left(u^{\prime}\right) \mathrm{d} u^{\prime} .
\end{aligned}
$$

In formula (5),

$$
\begin{aligned}
A_{\alpha} & =\frac{\exp (-j \pi \operatorname{sgn}(\sin \alpha) / 4+j \alpha / 2)}{|\sin \alpha|^{1 / 2}}, \\
\alpha & =\frac{p \pi}{2} .
\end{aligned}
$$

When the order $p \varepsilon[1,-1]$, formula (6) is decomposed into the calculation process of the following formulas:

$$
\begin{aligned}
& g\left(u^{\prime}\right)=\exp \left[-j \pi u^{\prime 2} \tan \left(\frac{\alpha}{2}\right)\right] x\left(u^{\prime}\right), \\
& g^{\prime}(u)=A_{\alpha} \int_{-\infty}^{\infty} \exp \left[j \pi \beta\left(u-u^{\prime}\right)^{2}\right] g\left(u^{\prime}\right) \mathrm{d} u^{\prime}, \\
& X_{p}(u)=\exp \left[-j \pi u^{2} \tan \left(\frac{\alpha}{2}\right)\right] g^{\prime}(u) .
\end{aligned}
$$

Here, $g\left(u^{\prime}\right)$ and $g^{\prime}(u)$ are just two intermediate results $\beta=\csc \alpha,-\pi / 2 \leq \alpha \leq \pi / 2$. Discretize equations (7)-(9) to obtain the numerical calculation method of discrete fractional Fourier transform [9].

2.3. Fractional Wavelet Transform. The scale factors $a=a_{0}^{k}$, $k \in z$ (where $a_{0}>1$ ) and the time shift factor $\Delta b=a_{0}^{k} b_{0}$ in the continuous fractional wavelet transform expression are discretized and sampled in the displacement domain, and the value corresponding to the sampling point can be expressed by the discrete fractional wavelet transform formula.

Discretize the scale factors $a=a_{0}^{k}, k \in z$ to get 


$$
\begin{aligned}
\psi_{p: k, n}(t) & =e^{-j t^{2}-\left(n a_{0}^{k} b_{0}\right)^{2} / 2 \cot \alpha} \frac{1}{a_{0}^{k}} \psi\left(\frac{t-n a_{0}^{k} b_{0}}{a_{0}^{k}}\right) \\
& =e^{-j j t^{2}-\left(n a_{0}^{k} b_{0}\right)^{2} / 2 \cot \alpha} a_{0}^{-k / 2} \psi\left[a_{0}^{-k}\left(t-n a_{0}^{k} b_{0}\right)\right] \\
& =e^{-j t^{2}-\left(n a_{0}^{k} b_{0}\right)^{2} / 2 \cot \alpha} a_{0}^{-k / 2} \psi\left[a_{0}^{-k} t-n b_{0}\right] .
\end{aligned}
$$

When $a=a_{0}^{0}=1$, the expression of discrete fractional wavelet transform is

$$
\begin{aligned}
\operatorname{DFRWT}_{f}(k, n) & =\left\langle f(t), \psi_{p: k, n}(t)\right\rangle=\int_{-\infty}^{+\infty} f(t) \psi_{p: k, n}(t) \mathrm{d} t \\
& =2^{-k / 2} \int_{-\infty}^{+\infty} f(t) e^{j t^{2}-\left(n 2^{k}\right)^{2} / 2 \cot \alpha} \psi\left[2^{-k}(t-n)\right] \mathrm{d} t .
\end{aligned}
$$

The reconstruction of the fractional wavelet transform is the inverse process of the decomposition process of the fractional wavelet. In the known $k$-th layer, the fractional wavelet coefficients are $\left\{c_{m}^{\prime k}\right\}_{m \in z}$ and $\left\{d_{m}^{\prime k}\right\}_{m \in z}$, and the original signal is $\left\{c_{n}^{0}\right\}$ through the reconstruction. $\left\{V_{k}^{\alpha}\right\}_{k \in z}$ is the multiresolution analysis, which can be seen from the relationship between $\psi_{p: k, n}(t), \phi_{p: k, n}(t)$, and the function projection:

$$
\begin{aligned}
& c_{n}^{\prime k+1}=c_{n}^{k+1} e^{-i 1 / 2\left(n \cdot 2^{k+1}\right)^{2} \cot \alpha} \\
& =\left\langle f(t), \phi_{p: k+1, n}(t)\right\rangle \\
& =\left\langle f_{k}(t), \phi_{p: k+1, n}(t)\right\rangle+\left\langle w_{k}(t), \phi_{p: k+1, n}(t)\right\rangle \\
& =\sum_{m \in z} c_{m}^{\prime k}\left\langle\phi_{p: k, m}(t), \phi_{p: k+1, n}(t)\right\rangle+\sum_{m \in z} d_{m}^{\prime k}\left\langle\psi_{p: k, m}(t), \phi_{p: k+1, n}(t)\right\rangle \\
& =\sum_{m \in z} c_{m}^{\prime k} e^{j 1 / 2\left(m 2^{-k}\right)^{2} \cot \alpha} e^{-j 1 / 2\left(n 2^{-(k+1)}\right)^{2} \cot \alpha}\left\langle\phi_{k, m}(t), \phi_{k+1, n}(t)\right\rangle+\sum_{m \in z} d_{m}^{\prime k} e^{j 1 / 2\left(m 2^{-k}\right)^{2} \cot \alpha} e^{-j 1 / 2\left(n 2^{-(k+1)}\right)^{2} \cot \alpha}\left\langle\psi_{k, m}(t), \phi_{k+1, n}(t)\right\rangle \\
& =\sum_{m \in z} c_{m}^{\prime k} e^{j 1 / 2\left(m 2^{-k}\right)^{2} \cot \alpha}\left\langle\phi_{k, m}(t), \phi_{k+1, n}(t)\right\rangle+\sum_{m \in z} d_{m}^{\prime k} e^{j 1 / 2\left(m 2^{-k}\right)^{2} \cot \alpha}\left\langle\psi_{k, m}(t), \phi_{k+1, n}(t)\right\rangle .
\end{aligned}
$$

Hence,

$$
\begin{aligned}
c_{n}^{k+1} & =\sum_{m \in z} c_{m}^{k}\left\langle\phi_{k, m}(t), \phi_{k+1, n}(t)\right\rangle+\sum_{m \in z} d_{m}^{k} e^{i(1 / 2)\left(n 2^{j+1}\right)^{2} \cot \alpha}\left\langle\psi_{k, m}(t), \phi_{k+1, n}(t)\right\rangle \\
& =2^{-1 / 2}\left[\sum_{m \in z} c_{m}^{k} h_{0}(n-2 m)+\sum_{m \in z} d_{m}^{k} h_{1}(n-2 m)\right] .
\end{aligned}
$$

Equation (13) is the reconstruction process of traditional discrete wavelet coefficients. Firstly, the fractional coefficients $c_{m}^{\prime k}$ and $d_{m}^{\prime k}$ of the $k$ layers are modulated, and then onedimensional wavelet inverse transformation is performed in the wavelet domain to obtain $c_{n}^{k+1}$, and then $c_{n}^{k+1}$ is modulated into the fractional wavelet domain to obtain $c_{n}^{\prime k+1}$, and so on, to restore the original signal $\left\{c_{n}^{0}\right\}$ step by step.

\subsection{Realization Process of Fractional Wavelet Transform.} With a one-dimensional signal $f(x)$, using the definition of fractional wavelet transform proposed by Menlovevic, the realization process of one-dimensional fractional wavelet transform can be obtained as follows:

(1) Input one-dimensional signal $f(x)$

(2) Select the appropriate fractional order change range $p$, and use the minimum output energy to search for the best transformation order

(3) Perform $p$-order fractional Fourier transform on the input signal $f(x)$ to obtain a signal in the fractional domain 
(4) Perform wavelet decomposition on the obtained signal in the fractional domain to obtain a signal in the fractional wavelet domain

(5) Perform wavelet reconstruction on the signal in the fractional wavelet domain to recover the signal in the fractional domain

(6) Perform - p-order fractional Fourier transform on the signal in the fractional domain to recover the filtered signal $[10,11]$

The realization process of fractional wavelet transform is shown in Figure 1 [8].

\section{Wavelet Energy and Two-Dimensional Convolutional Neural Network}

\subsection{Feature Extraction Process Based on Wavelet Energy.}

The main steps are as follows:

(1) The signal is decomposed by $n$-layer wavelet packet, the $j$-th layer has $2^{n}$ frequency band signals, and then $2^{n}$ features of the $n$-th layer are extracted.

(2) In order to improve the denoising ability of the signal, select the low-frequency coefficients and high-frequency coefficients of each frequency band decomposed in (1) to reconstruct the signal, denoted as $f$.

(3) Solve the energy $E_{i, j}$ of each signal, and the calculation formula for the energy value of each frequency band is as follows:

$E_{i, j}\left(t_{j}\right)=\int\left|f_{i, j}\left(t_{j}\right)\right|^{2} \mathrm{~d} t=\sum_{k=1}^{m}\left|x_{j, k}\right|^{2} \quad j=0,1,2, \ldots, 2^{i}-1$.

Here, $x$ is the decomposition coefficient of the wavelet packet, $E_{i, j}\left(t_{j}\right)$ is the energy value of the $j$-th node in the $i$-th layer after the signal $x(t)$ undergoes wavelet decomposition, $k=1,2, \ldots, N_{c}$, and $x_{i, k}$ is the wavelet packet reconstruction coefficient of $f_{i, j}$ [12].

(4) Construct feature vector.

In the process of wavelet decomposition, the energy of each layer is equal to the total energy, and the total energy of the signal is

$$
\begin{aligned}
E_{\text {sum }} & =E_{i} \\
& =\sum_{j} E_{i, j} .
\end{aligned}
$$

The wavelet packet energy of each frequency band is

$$
M_{i, j}=\frac{E_{i, j}}{E_{\text {sum }}} .
$$

The wavelet packet energy feature vector is

$$
W_{n}=\left(M_{n, 0}, M_{n, 1}, \ldots, M_{n, 2^{n}-1}\right) .
$$

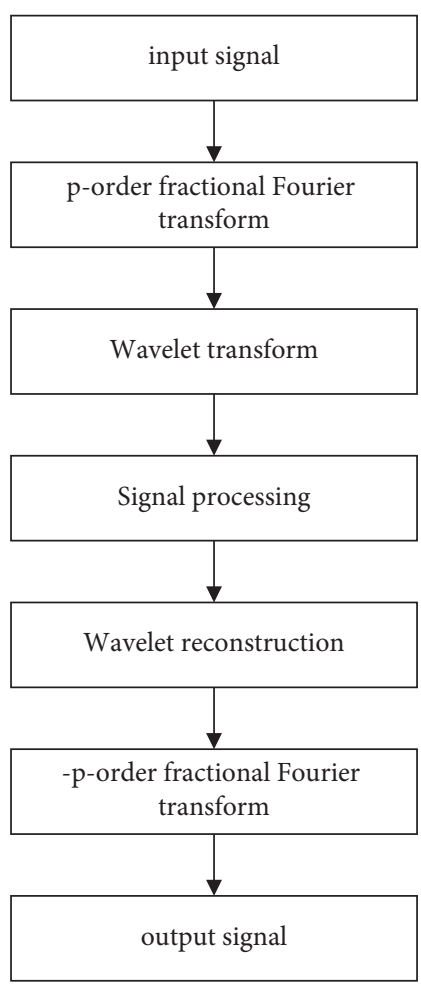

Figure 1: The basic process of fractional wavelet transform.

3.2. Structure of a Two-Dimensional Convolutional Neural Network. The current typical two-dimensional convolutional neural network structure is composed of input layer, convolution layer, pooling layer, fully connected layer, and output layer. The network structure of LeNet -5 is shown in Figure 2. The input of the convolutional neural network is mainly in the form of a two-dimensional grayscale image or a color image. Its output layer uses the Softmax classifier to output the classification and recognition results of a twodimensional grayscale image or a color image. In other image processing fields such as target detection, other forms of network output layers need to be set up $[13,14]$.

The convolutional layer is composed of multiple convolutional neurons. The parameters of the convolutional neuron are obtained by using the backpropagation algorithm. The convolutional layer is a key part of the entire convolutional neural network, which is mainly used for input data to extract different features [15]; the process of convolution operation is composed of continuous convolution and discrete convolution.

The process of discrete convolution operation is as follows:

$$
y(n)=x(n) * h(n)=\sum_{i=0}^{N-1} x(i) h(n-i)
$$

When the image convolution operation is performed, it is the operation between the image pixels. The pixels of the image can be understood as a matrix, and the pixels are not continuous. The process of the convolution operation is the selected convolution kernel and the image. Input for 


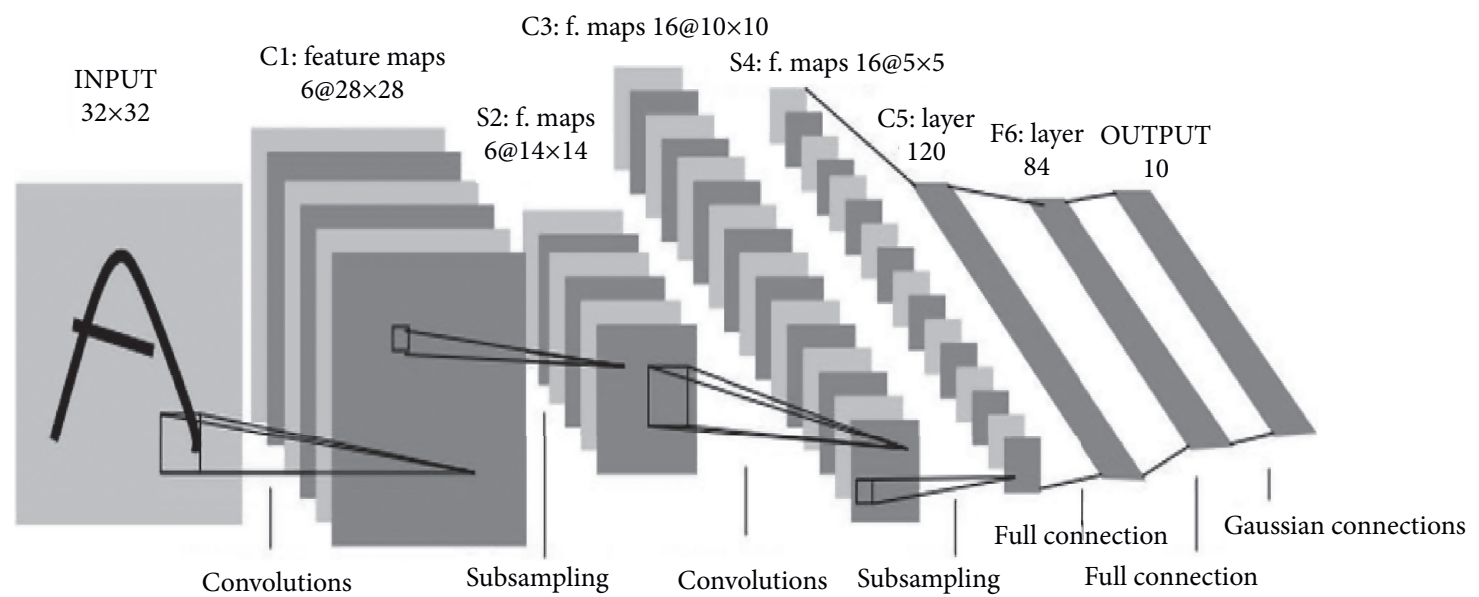

Figure 2: Schematic diagram of LeNet-5 structure.

convolution operation: Assuming that the two-dimensional image input is $I(i, j)$ and the two-dimensional convolution kernel is $K(m, n)$, the image convolution operation process can be expressed as

$$
S(i, j)=(I * K)(i, j)=\sum_{m} \sum_{n} I(m, n) K(i-m, j-n) .
$$

Convolution operation is alternating, so

$$
S(i, j)=(K * I)(i, j)=\sum_{m} \sum_{n} I(i-m, j-n) K(m, n) .
$$

Here, $m, n$ is the size of the convolution kernel. After the feature is extracted by the convolution operation, the offset operation needs to be performed after the convolution operation. The calculation formula is as follows:

$$
x_{j}^{i}=f\left(\sum_{i \in M_{j}} x_{j}^{i-1} * k_{i j}^{l}+b_{j}^{l}\right) .
$$

Here, $x_{j}^{i}$ is the first feature map output by the first layer; $f(x)$ is the activation function used by the convolutional layer; $k_{i j}^{l}$ is the convolution matrix used by the convolution kernel; and $b$ is the offset of the convolution operation.

The pooling layer is also commonly referred to as the downsampling layer. The pooling layer can reduce the training time of the model, improve the robustness of feature extraction, and avoid overfitting of the model. There are usually three ways of pooling: average pooling process, maximum pooling process, and random pooling process. In actual applications, the pooling process is dominated by maximum pooling.

Maximum pooling calculation formula is

$$
p_{i}=\operatorname{Max}_{k \in M_{j}}\left\{a_{i}(k)\right\} .
$$

In the actual application process, the classifier needs to be trained in the fully connected layer. The commonly used classifier is the Softmax classifier. The fully connected process is shown in the following formula:

$$
y^{k}=f\left(w^{k} x^{k-1}+b^{k}\right) \text {. }
$$

In formula (23), $y^{k}$ is the output of the fully connected layer; $w^{k}$ is the weight value; $x^{k-1}$ is the input of the fully connected layer; $b^{k}$ is the bias term; $f(x)$ is the classification function; $k$ is the network layer number.

In image classification, Softmax is generally used as the classifier. If there are $K$ classifications, the output of Softmax can be expressed as

$$
\sigma(x)_{i}=\frac{e^{z i}}{\sum_{j}^{k} e^{z j}}, \quad i=1,2, \ldots, k .
$$

3.3. Procedure. In order to accurately classify planetary gear faults in a complex actual industrial environment, this paper proposes a planetary gear fault diagnosis method based on FRWT and 2D-CNN. A flowchart can be drawn as shown in Figure 3.

The specific steps are as follows:

(1) Use fractional wavelet transform to separately denoise the gear fault signals

(2) Use Shannon entropy to extract energy from the signal after noise reduction and calculate the normalized energy value

(3) Convert the obtained wavelet energy value into a two-dimensional matrix feature sample set

(4) Initialize the two-dimensional convolutional neural network and use the sample set to extract the characteristics of the signal

(5) Train and establish a two-dimensional convolutional neural network model to identify planetary gear faults

\section{Experimental Verification}

4.1. Introduction to the Experimental Sample Set. The planetary gear fault experiment data used in this article is collected by the QPZZ-II mechanical fault simulation and test platform produced by Jiangsu Qianpeng Diagnostic Engineering Co., Ltd. The test platform includes drive motors, 


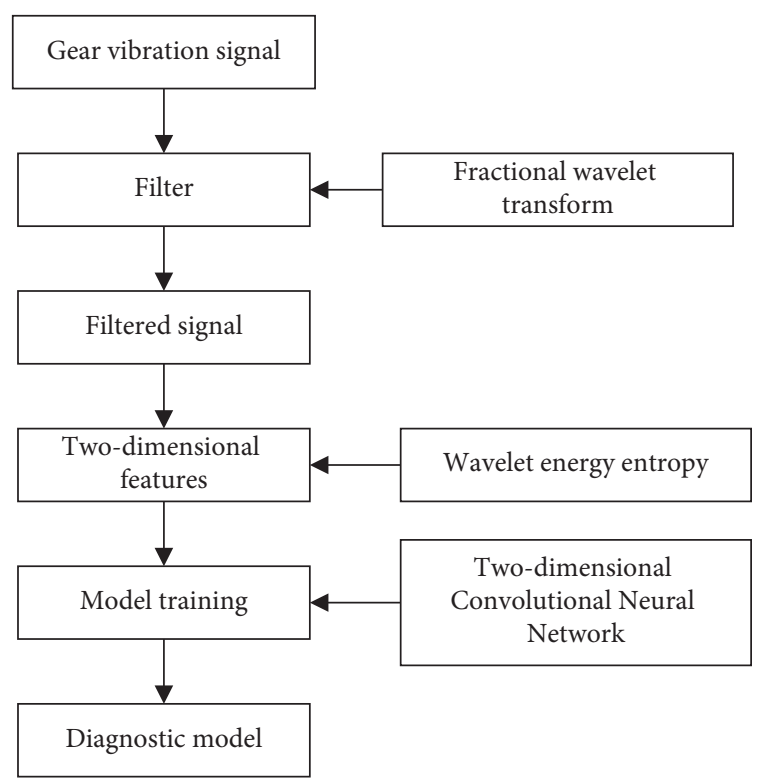

FIgURE 3: Fault diagnosis flowchart of planetary gear.

planetary gears, rolling bearings, speed control equipment, etc. The fault components used in the entire test platform include planetary gear pitting fault, broken tooth fault, wear fault, and other faults. The sampling frequency of the experimental data is $5120 \mathrm{~Hz}$. A total of 10 fault samples under fault states were selected, 1700 samples were selected for each fault, and the number of sample points for each sample was 512. Among them, the ratio of the number of training samples to the number of test samples is $10: 7$, and the specific sample classification is shown in Table 1.

\subsection{FRWT Filter Analysis}

4.2.1. Determination of the Optimal Order of FRWT. First, the minimum output energy is used as the objective function to optimize the optimal order. The order optimization process of pitting fault reconstruction signal, broken tooth fault reconstruction signal, and wear fault reconstruction signal is shown in Figure 4. It can be clearly seen from Figure 4 that the minimum value of the FRFT output energy of the pitting fault (Dianshi880-1) reconstructed signal is 11670, and the corresponding order is 1.57; that is, the best order is 1.57; in the broken tooth fault (Duanchi1500) the minimum value of the FRFT output energy of the reconstructed signal is 20020, and the corresponding order is 1.646; that is, the best order is 1.646; the minimum value of the FRFT output energy of the reconstructed signal FRFT for wear fault (Mosun880-1) is 50360. At this time, the corresponding order is 1.558; that is, the best order is 1.558 . The output energy value and the corresponding optimal order of the remaining faults are shown in Table 2.

4.2.2. Determination of the Number of FRWT Wavelet Bases and Decomposition Layers. In the fractional wavelet transform, when the selected wavelet base and the number of decomposition layers are different, the noise reduction effect of the signal will be different. Therefore, the wavelet bases are selected as $\mathrm{db} 1 \sim \mathrm{db} 4$ and sym $1 \sim \operatorname{sym} 4$, respectively, and the number of decomposition levels is 1 to 5 , and the optimal wavelet base and decomposition level are selected by calculating the output signal-to-noise ratio (SNR) of the denoising signal. The specific results are shown in Figure 5-Figure 7. The wavelet basis and decomposition layer settings for each fault are shown in Table 3.

4.2.3. FRWT and FRFT Filtering Effect Analysis. The pitting fault reconstruction signal, wear fault reconstruction signal, and broken tooth fault reconstruction signal are, respectively, subjected to FRFT filtering and FRWT filtering, and the filtering results of each fault signal are shown in Figure 8, Figure 9, and Figure 10. Using the found optimal fractional order $p=1.57,1.646$, and 1.558, the pitting reconstruction signal, broken tooth reconstruction signal, and wear reconstruction signal are, respectively, subjected to fractional Fourier transform filtering. The filtering results are shown in Figure 8(b), as shown in Figure 9(b) and Figure 10(b), and then perform wavelet transform on the signal after the fractional Fourier transform in the corresponding fractional domain and finally carry out the transformed signal, $p$ $=-1.57,-1.646,-1.558$-order fractional Fourier transform to obtain the corresponding output signal time domain diagram as shown in Figure 8(c), Figure 9(c), Figure 10(c).

In order to analyze the influence of the fractional order on the signal filtering effect, this paper calculates the output signal-to-noise ratio of the two filtering methods, respectively. The input signal-to-noise ratio of the pitting fault (Dianshi880-1) signal is $-12.25 \mathrm{~dB}$; the broken tooth fault (the input signal-to-noise ratio of Duanchi1500) signal is $-13.15 \mathrm{~dB}$; the input signal-to-noise ratio of wear fault (Mosun880-1) signal is $-16.47 \mathrm{~dB}$. The comparison result is shown in Figure 11.

It can be seen from Figure 11 that the output signal-tonoise ratios (SNR) of FRWT for pitting faults, wear faults, and broken teeth faults are all greater than the output signalto-noise ratio (SNR) of FRFT. According to the larger output signal-to-noise ratio (SNR), the signal will be distorted. The smaller the degree and the noise interference, the better the filtering effect of FRWT compared to the filtering effect of FRFT.

4.3. Wavelet Packet Extraction Features. Set the decomposition level of the wavelet packet to 8 , which will generate a total of 256 frequency bands, and use the wavelet basis $d b 3$ to decompose the fault vibration signals of 10 gears into eight layers, and generate a total of 256 wavelet packet components. Then use Shannon entropy to extract the wavelet energy, and then process the energy of the frequency band, that is, obtain the sum of the norm squares of each node of each layer of neurons, and finally obtain the normalized energy amplitude of each node. The corresponding normalized energy value of each frequency band is shown in Figure 12. 
TABLE 1: Sample set.

\begin{tabular}{lcccc}
\hline Data set name & Fault type & Number of training set samples & Number of test machine samples & Label \\
\hline Mosun880 & Wear & 1000 & 700 & 700 \\
Mosun880-1 & Wear & 1000 & 700 & 2 \\
Mosun880-2 & Wear & 1000 & 700 & 3 \\
Mosun880-3 & Wear & 1000 & 700 & 4 \\
Dianshi880 & Pitting & 1000 & 700 & 7 \\
Dianshi880-1 & Pitting & 1000 & 700 & 7 \\
Dianshi880-2 & Pitting & 1000 & 700 & 9 \\
Dianshi880-3 & Pitting & 1000 & 100 & 9 \\
Duanchi1500 & Broken tooth & 1000 & 700 & 10 \\
Normal880 & Normal & 1000 & & \\
\hline
\end{tabular}
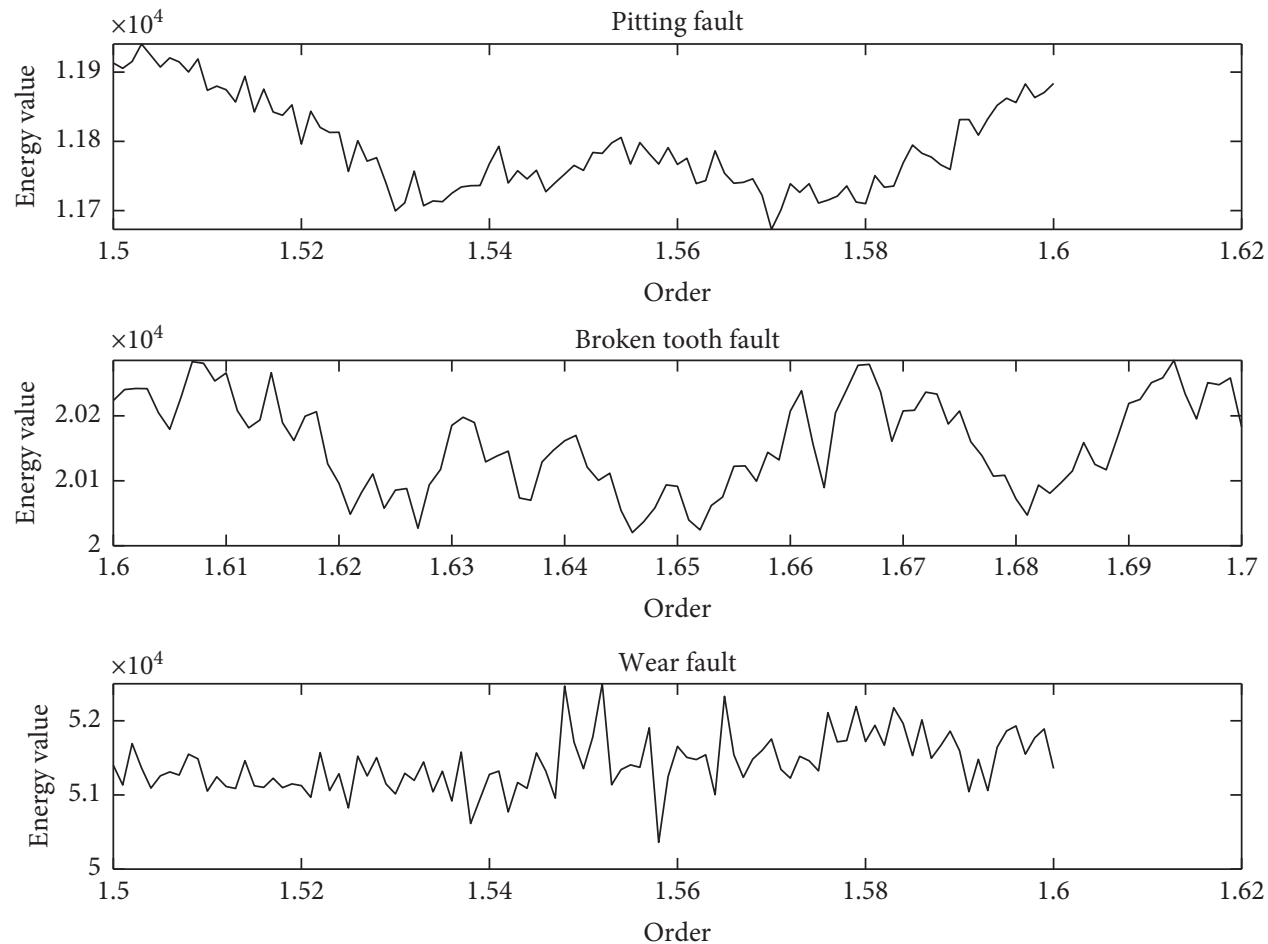

FIGURE 4: FRWT order optimization.

TABLE 2: Fault input signal-to-noise ratio, FRWT optimal order, and energy value.

\begin{tabular}{lccc}
\hline Fault type & Enter SNR $(\mathrm{db})$ & Best order & Minimum energy \\
\hline Mosun880 & -13.58 & 1.1 & 35250 \\
Mosun880-2 & -20.65 & 1.42 & 62135 \\
Mosun880-3 & -25.47 & 1.25 & 70259 \\
Dianshi880 & -10.02 & 1.291 & 9875 \\
Dianshi880-2 & -15.63 & 1.432 & 13026 \\
Dianshi880-3 & -20.87 & 1.45 & 16548 \\
Normal880 & -10.25 & 1.31 & 7458 \\
Dianshi880-2 & -15.63 & 1.432 & 13026 \\
\hline
\end{tabular}

Among them, the characteristics of each sample are 256 frequency band energy spectra, and the characteristics of 1700 samples are converted into a matrix form with a twodimensional form with a dimension of $16 * 16$. Figure 13 shows the converted two-dimensional frequency band energy characteristic distribution. Finally, the One - hot code is used to set the label category for each type of fault.
4.4. Training and Classification of Fault Models. The specific parameter selection for experimental verification is as follows: the number of layers of the two-dimensional CNN network is set to 6 layers, the convolutional layer and the pooling layer are each two layers, the number of convolution kernels in the first layer is 8 , and its size is $3 * 3$. The number of convolution kernels in the second layer is 16 , and its size is 

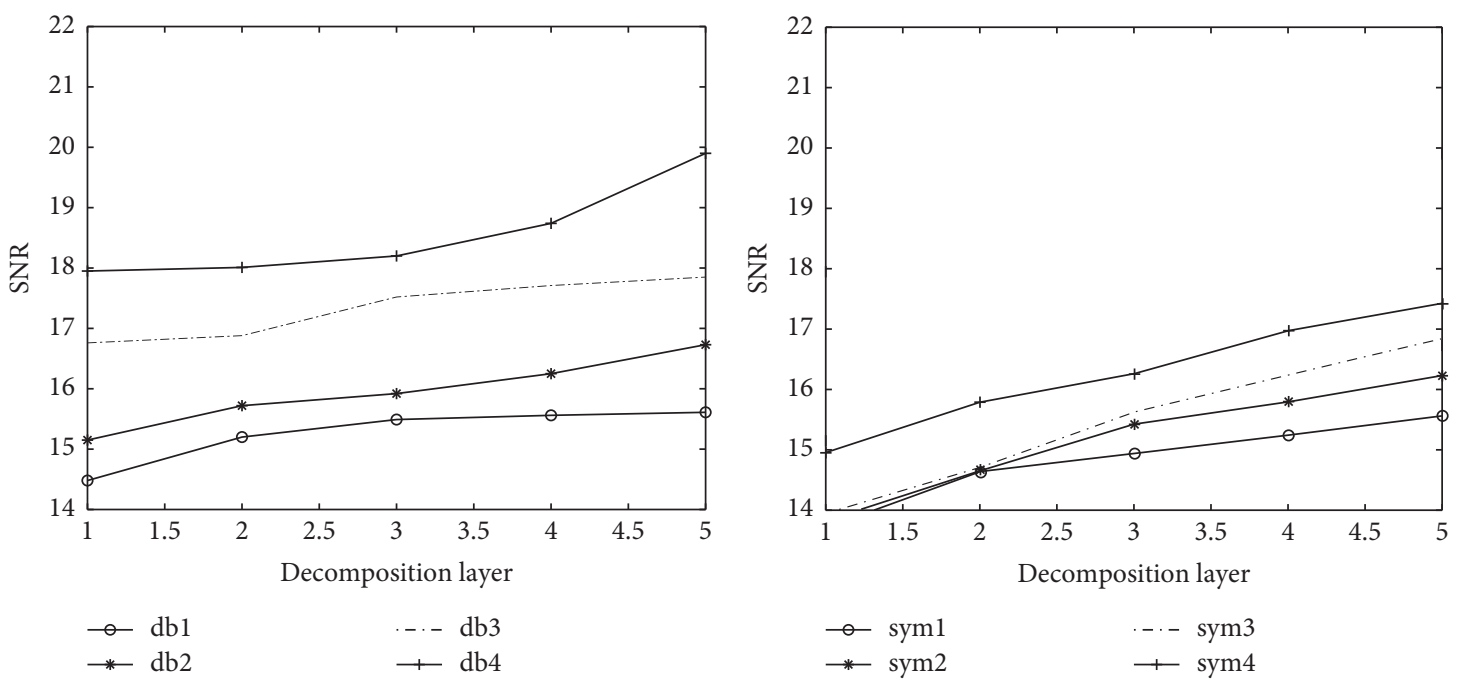

Figure 5: The relationship between the signal-to-noise ratio of the pitting signal and the wavelet basis and the number of decomposition layers.
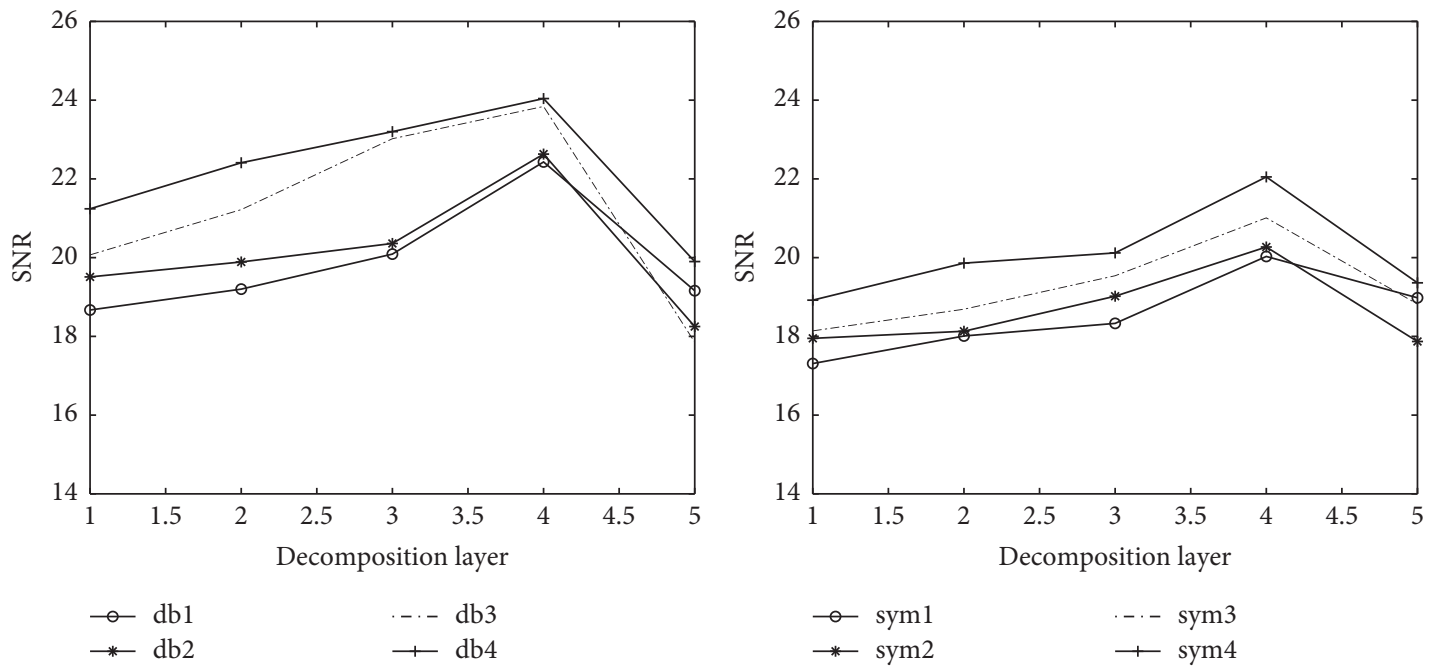

FIGURE 6: The relationship between the signal-to-noise ratio of the broken tooth signal and the wavelet basis and the number of decomposition layers.

$4 * 4$; the batch size is 10 , and the maximum number of iterations is 1500; the pooling layer uses the maximum pooling method, and its size is $2 * 2$; using Dropout regularization reduces overfitting. Extract the wavelet energy values of the signals after FRFT filtering and FRWT filtering to construct a two-dimensional feature matrix as input; randomly select 1000 samples of each type of fault as the training set for model training, and 700 samples as the test set for the two-dimensional convolutional neural. The training model of the network is verified, and the training error curve is shown in Figure 14.

From the analysis in Figure 14, it can be seen that, regardless of whether the fractional Fourier transform or the fractional wavelet transform is used, when the number of iterations is less than or equal to 120 , the training error of the two is equal; when the number of iterations is 120 , the training error is 0.6667 . The effect is extremely poor; when the number of iterations is greater than 120, the training error of the fractional wavelet transform filtering signal is obviously smaller than the training error of the fractional Fourier transform filtering signal; when the number of iterations is 1500 , the training error of the fractional wavelet transform filtering method is 0.01623 , and the training error of the fractional Fourier transform filtering method is 0.06514 , that is; the training error of the fractional wavelet transform filter signal is significantly smaller than the training error of the fractional Fourier transform filter signal. It can be seen that the training effect of FRWT+2D$\mathrm{CNN}$ is better than that of FRWT+2D-CNN.

The classification results of each fault in the test set using the FRWT+2D-CNN and FRFT+2D-CNN models are shown in Figure 15 and Figure 16. The abscissa is the 

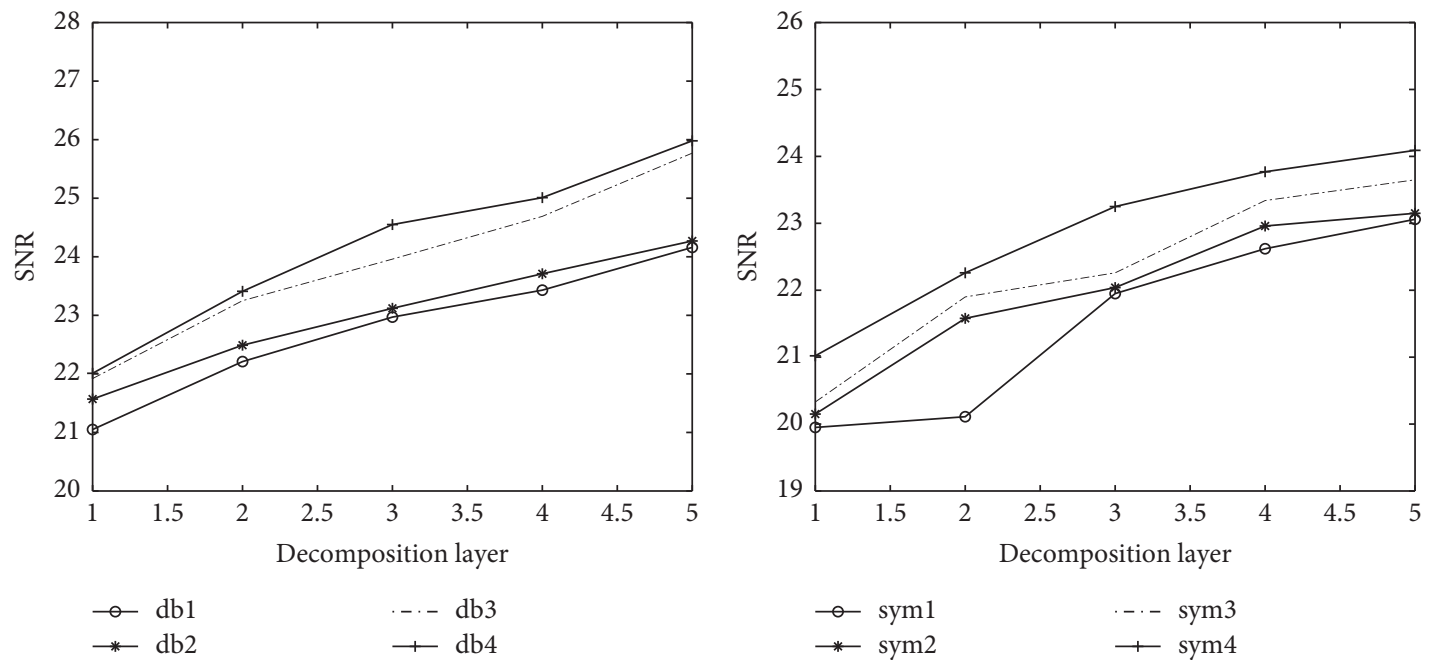

FIGURE 7: The relationship between the signal-to-noise ratio of the wear signal and the wavelet basis and the number of decomposition layers.

TABLE 3: Wavelet basis and decomposition layer settings for each fault.

\begin{tabular}{lcc}
\hline Fault type & Wavelet base & Decomposition layer \\
\hline Mosun880 & $\mathrm{db} 4$ & 4 \\
Mosun880-2 & $\mathrm{db} 4$ & 4 \\
Mosun880-3 & $\mathrm{db} 4$ & 4 \\
Dianshi880 & $\mathrm{db} 4$ & 5 \\
Dianshi880-2 & $\mathrm{db} 4$ & 5 \\
Dianshi880-3 & $\mathrm{db} 4$ & 5 \\
Normal880 & $\mathrm{db} 3$ & 4 \\
\hline
\end{tabular}

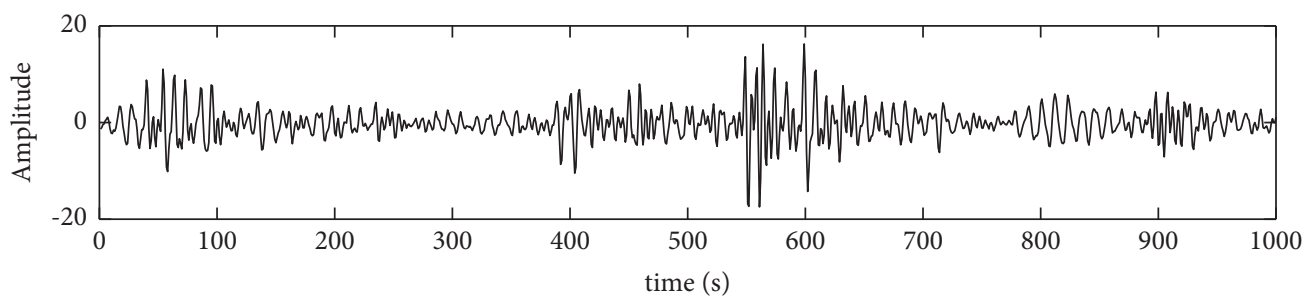

(a)

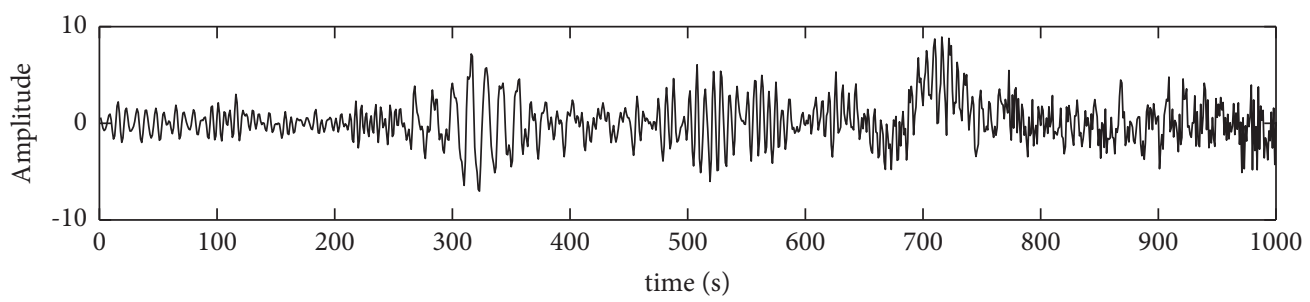

(b)

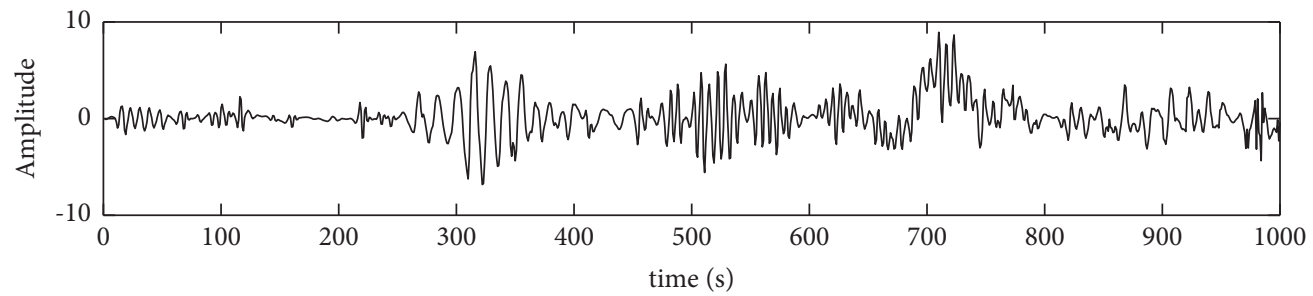

(c)

FIgURE 8: Time domain diagram of pitting signal filtering. (a) Pitting corrosion reconstruction signal. (b) FRFT filtered signal. (c) FRWT filtered signal. 


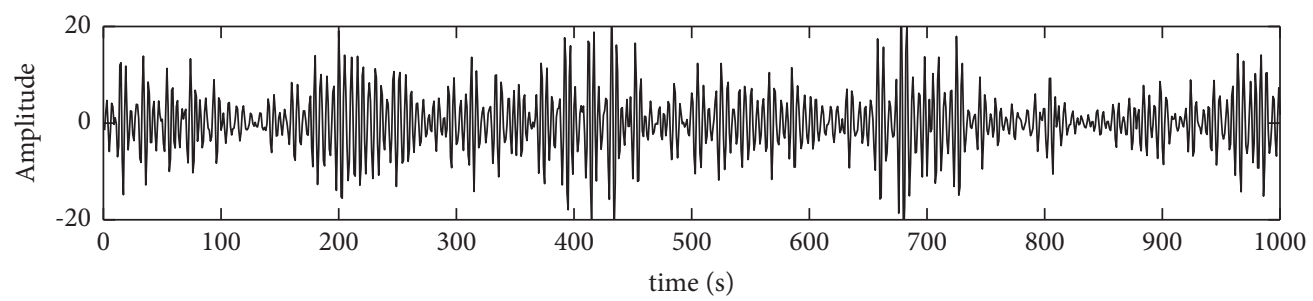

(a)

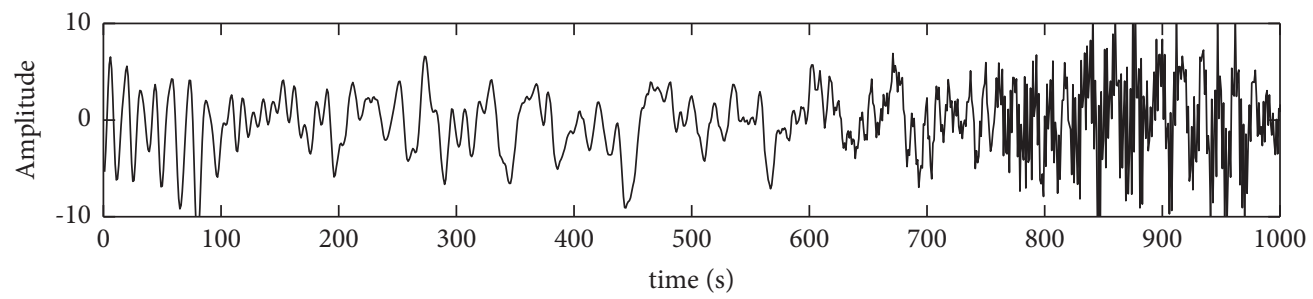

(b)

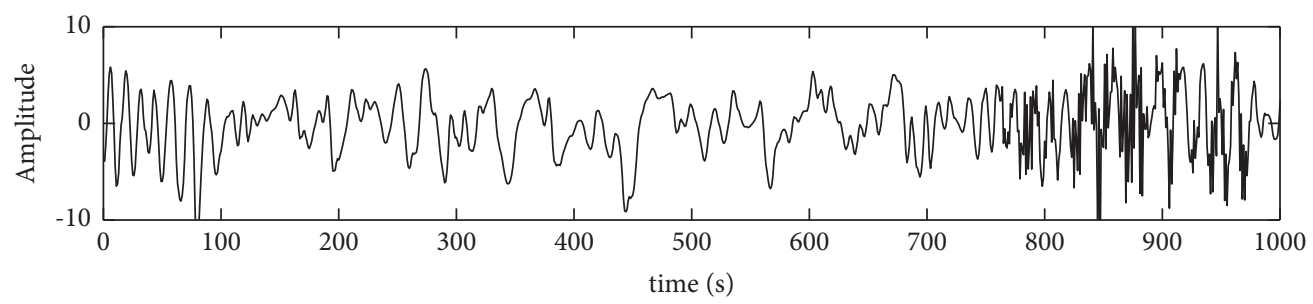

(c)

FIGURE 9: Time domain diagram of broken tooth signal filtering. (a) Tooth reconstruction signal. (b) FRFT filtered signal. (c) FRWT filtered signal.

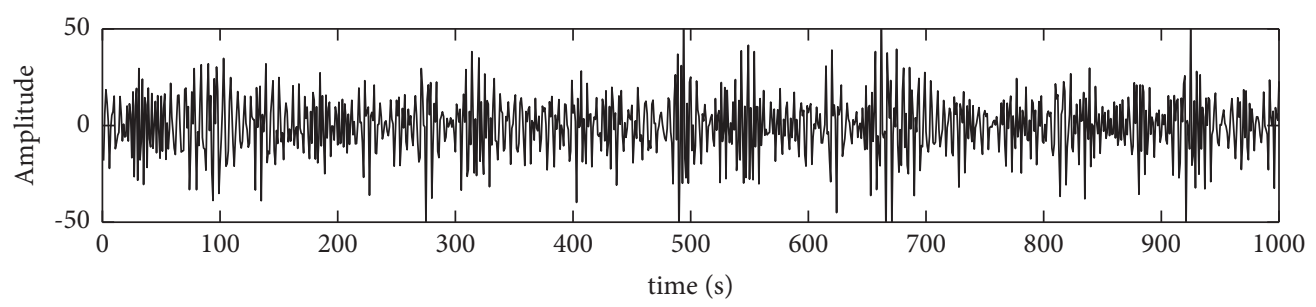

(a)

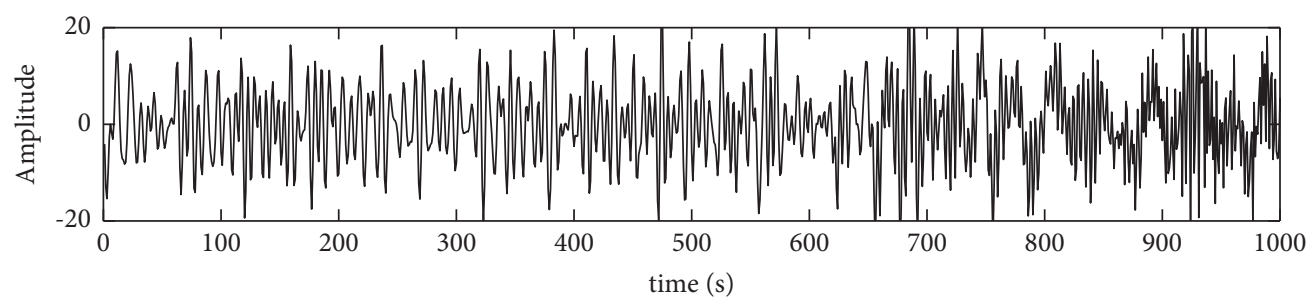

(b)

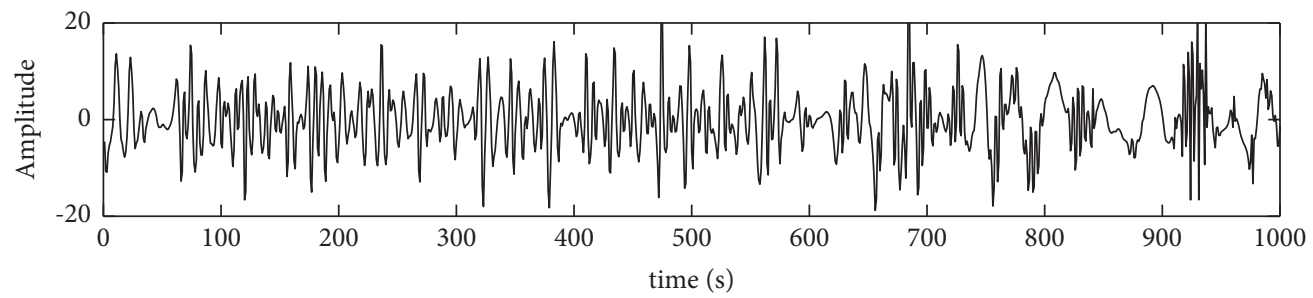

(c)

FIGURE 10: Time domain diagram of wear signal filtering. (a) Wear reconstruction signal. (b) FRFT filtered signal. (c) FRWT filtered signal. 


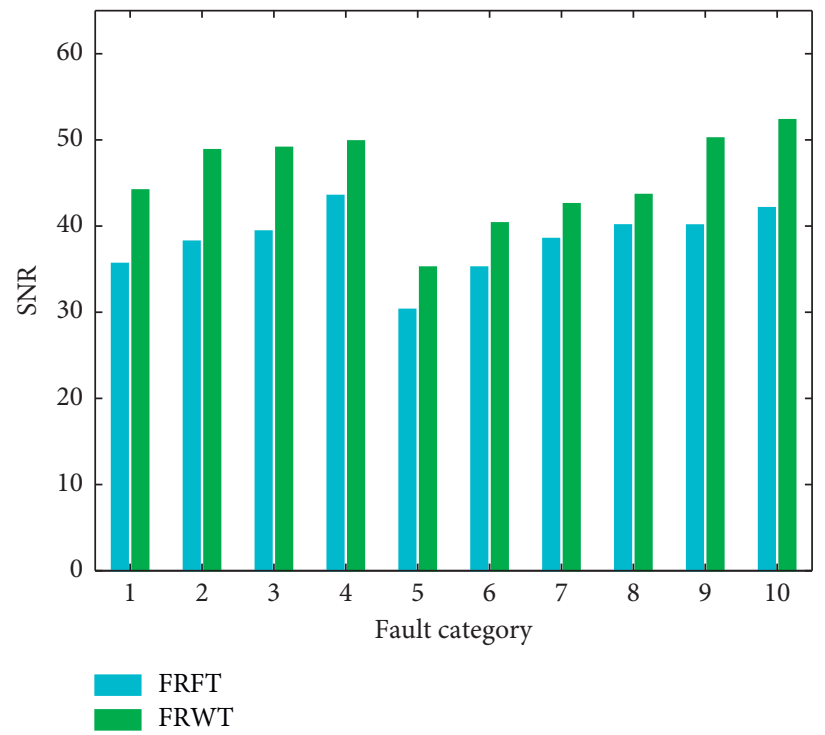

FIGURE 11: FRFT and FRWT filter output signal-to-noise ratio (SNR).
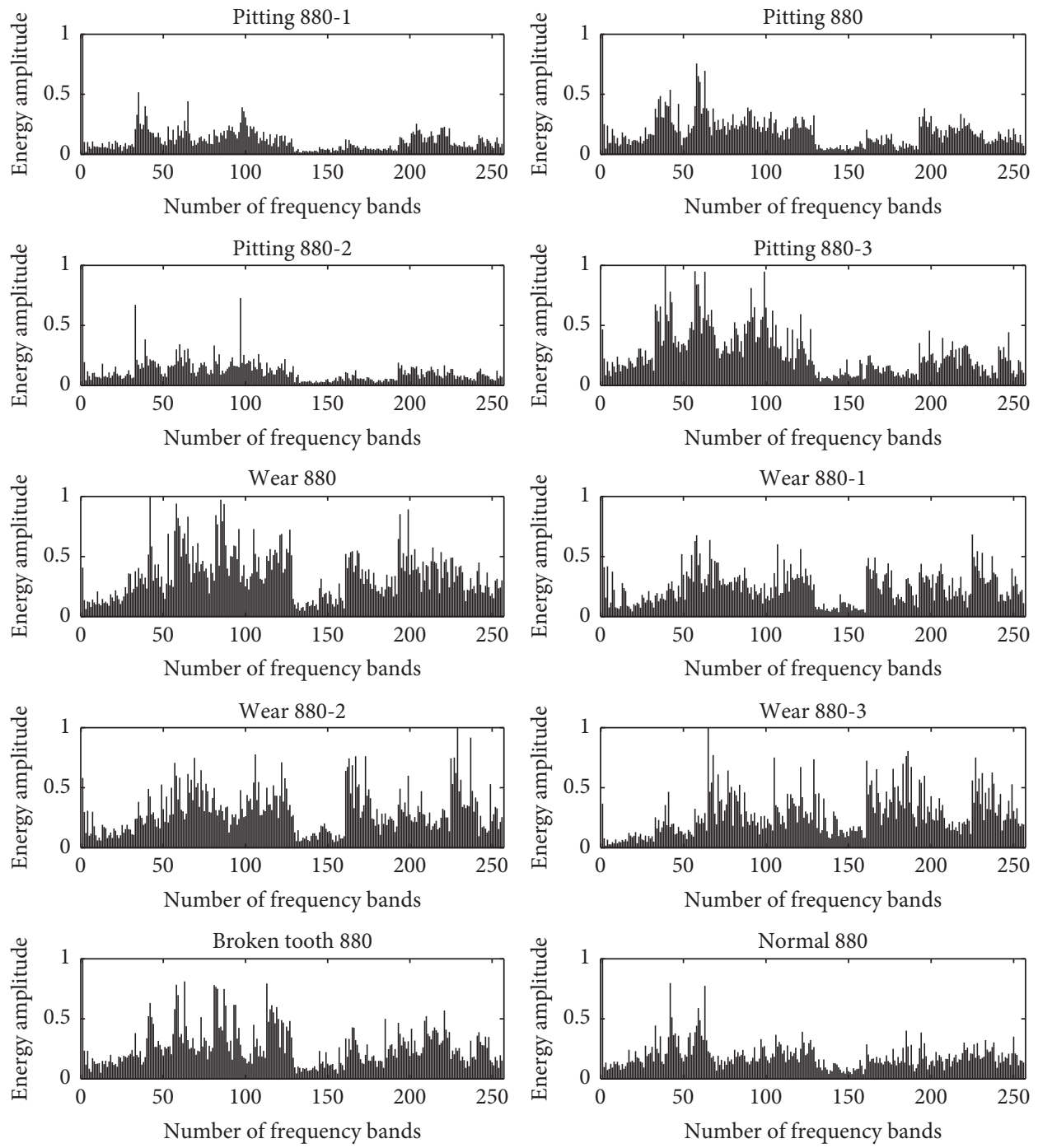

Figure 12: The relationship between the number of frequency bands of each fault and the normalized energy value. 

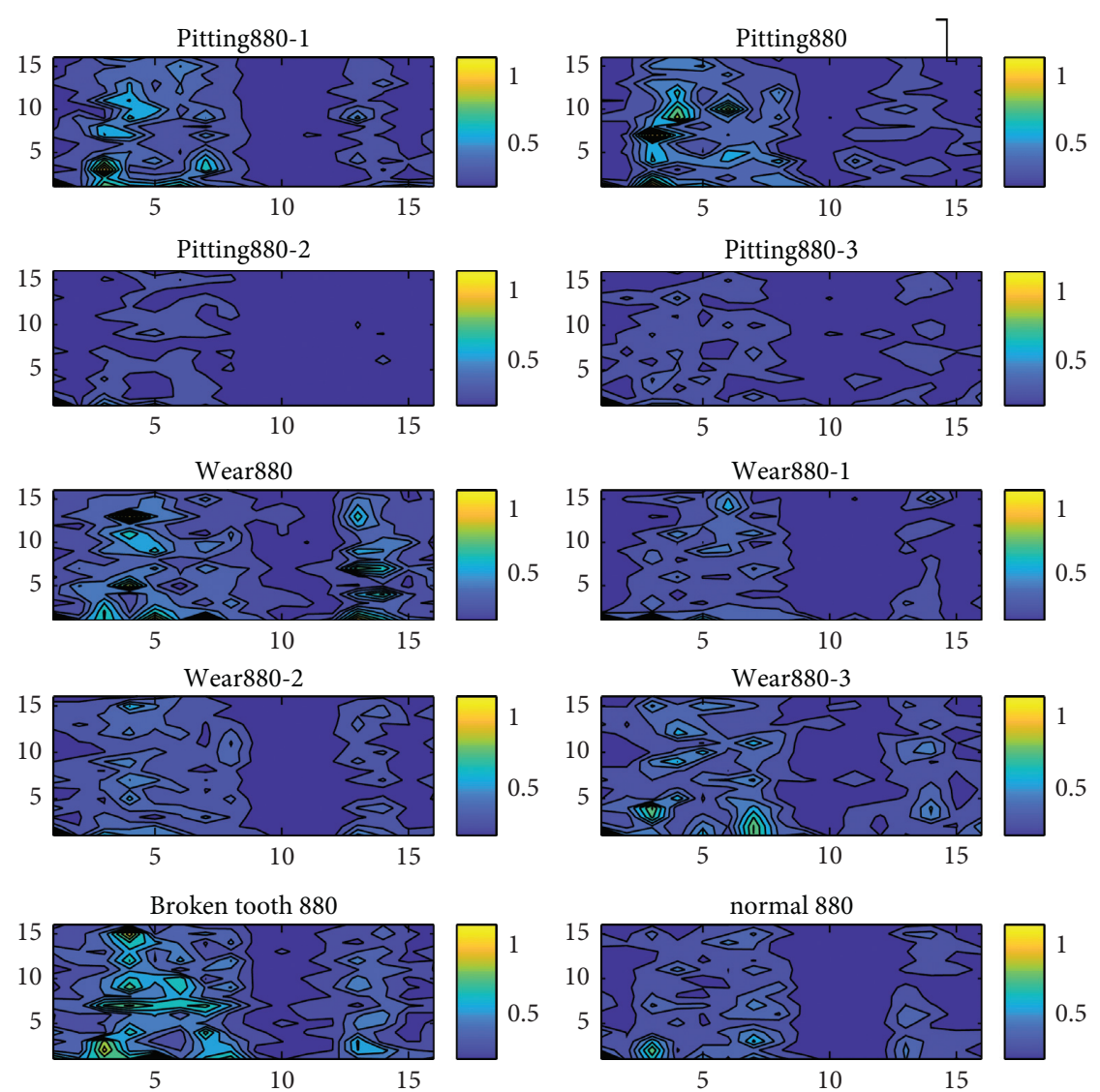

FIGURE 13: Feature distribution of some samples of the FRWT filtered signal.

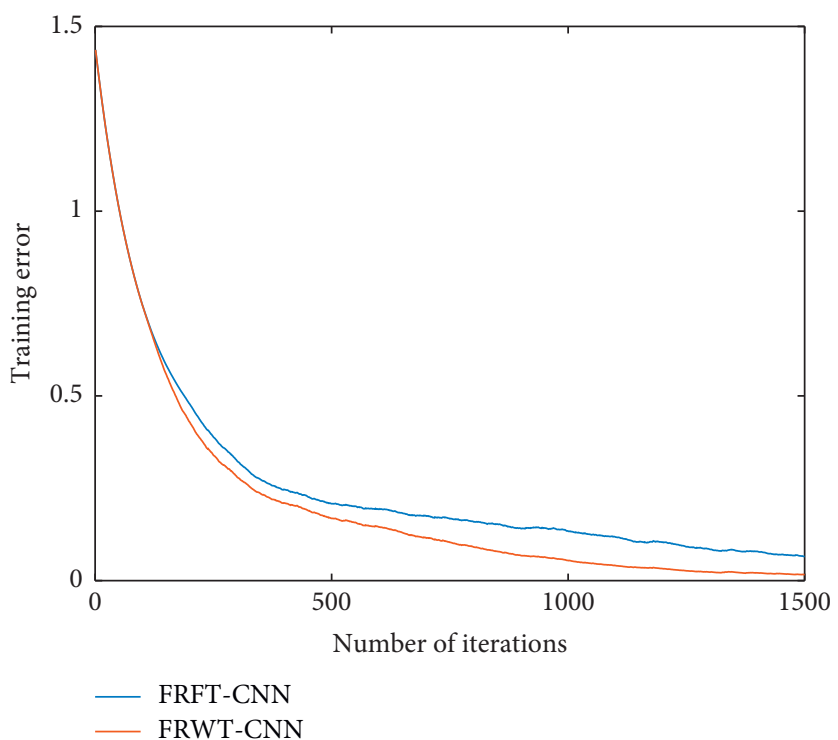

Figure 14: The relationship between model training error and training times.

predicted category label of the test set; the ordinate is the actual label category of the test set; the value of the diagonal position is the classification accuracy of each of the 10 types of faults; the position outside the diagonal is the type of fault.
Comparing Figures 15 and 16, it can be found that when FRWT+2D-CNN classifies and recognizes faults, only two samples are misclassified; that is, type 3 faults are misclassified as type 4 faults, and type 7 faults are wrong. The fault is classified as the 8th type of fault; when FRFT+2D- 


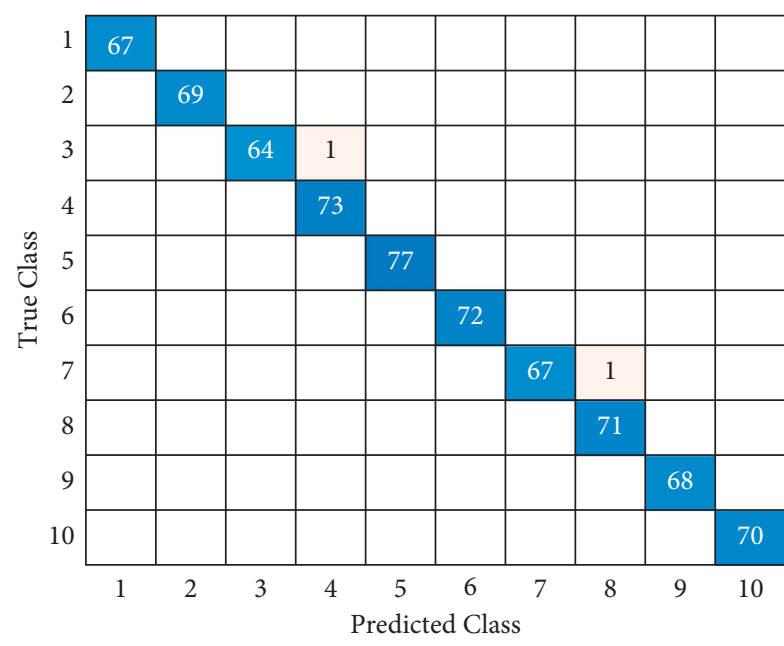

FIgURE 15: FRWT+2D-CNN test set classification label.

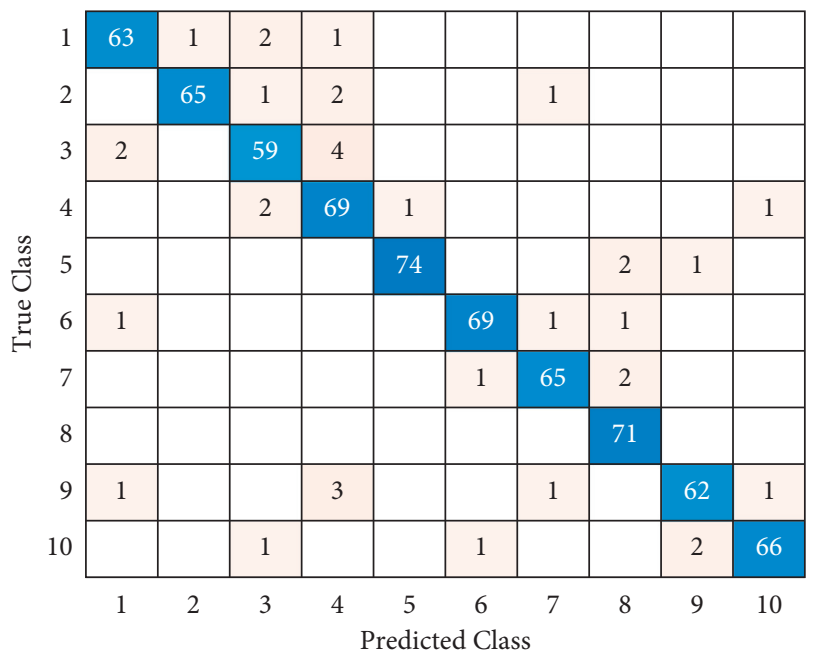

Figure 16: FRFT+2D-CNN test set classification label.

CNN classifies and recognizes the fault, except for the 5th type of fault, the other fault types are all misclassified, and the number of misclassified samples for each type of fault is greater than 1 . Therefore, when classifying faults based on FRWT+2D-CNN, each type of fault can be accurately identified to the greatest extent possible.

In order to fully verify the stability and accuracy of the diagnosis method proposed in this article, this article randomly conducts 15 simulation tests on the two diagnosis methods (FRFT+2D-CNN, FRWT+2D-CNN), and the classification accuracy of each test is as shown in Figure 17. The average accuracy of the diagnosis models of the two classification methods is shown in Table 4.

By analyzing Figure 5, 20, it can be seen that the classification accuracy of the two diagnostic methods FRFT+2D$\mathrm{CNN}$ and FRWT+2D-CNN basically remains stable, and the classification accuracies of FRFT+2D-CNN and FRWT+2D$\mathrm{CNN}$ are both within $3 \%$. With fluctuations up and down, from a macroperspective, the classification accuracy of

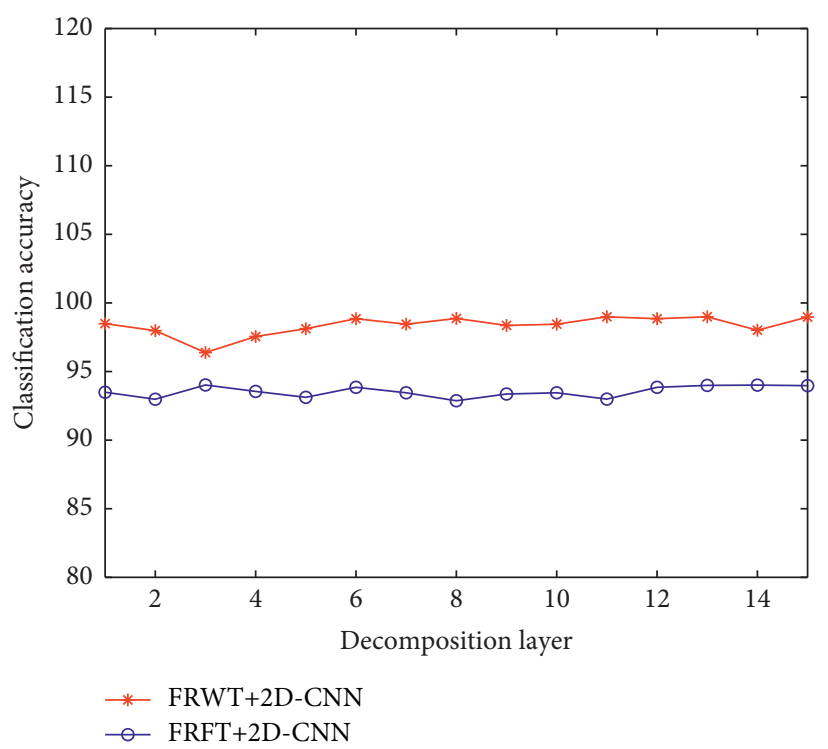

FIgURE 17: Test accuracy of the two methods.

TABLE 4: The average accuracy of classification by different methods.

\begin{tabular}{lcccc}
\hline $\begin{array}{l}\text { Diagnosis } \\
\text { method }\end{array}$ & $\begin{array}{c}\text { Training } \\
\text { samples }\end{array}$ & $\begin{array}{c}\text { Test } \\
\text { sample }\end{array}$ & $\begin{array}{c}\text { Number of } \\
\text { experiments }\end{array}$ & $\begin{array}{c}\text { Average } \\
\text { accuracy }\end{array}$ \\
\hline $\begin{array}{l}\text { FRFT+2D- } \\
\text { CNN }\end{array}$ & 1000 & 700 & 15 & 93.53 \\
$\begin{array}{l}\text { FRWT+2D- } \\
\text { CNN }\end{array}$ & 1000 & 700 & 15 & 98.36 \\
\hline
\end{tabular}

FRWT+2D-CNN is higher than that of FRFT+2D-CNN. It can be seen from Table 4 that when the number of training samples, the number of test samples, and the number of trials are equal, the average accuracy of FRWT $+2 \mathrm{D}-\mathrm{CNN}$ classification is higher than the average accuracy of FRFT+2D-CNN classification. Therefore, in the fault diagnosis of planetary gears, the classification method of FRWT+2D-CNN is obviously better than the fault classification method of FRFT+2D-CNN.

\section{Conclusion}

(1) This paper adopts the FRWT-based planetary gear vibration signal filtering method. The simulation results show that both the fractional wavelet transform and the fractional Fourier transform can achieve the denoising effect of the signal; the denoising effect of the fractional wavelet transform is better than fractional Fourier transform: The energybased fractional Fourier transform algorithm is better than the peak search-based fractional Fourier transform algorithm.

(2) This paper adopts a two-dimensional convolutional neural network model, and the signals after the fractional Fourier transform and the fractional wavelet transform are filtered, and the one-dimensional wavelet energy value is normalized and 
converted into a two-dimensional feature matrix for diagnosis model training. The simulation results show that the two-dimensional convolutional neural network can effectively realize fault classification and recognition. In addition, the accuracy of planetary gear fault classification based on FRWT and 2D$\mathrm{CNN}$ is better than the accuracy of planetary gear fault classification based on FRFT and 2D-CNN.

\section{Data Availability}

The data used to support the findings of this study are available from the corresponding author upon request.

\section{Conflicts of Interest}

The authors declare that there are no conflicts of interest regarding the publication of this paper.

\section{Acknowledgments}

This work was supported by the National Natural Science Foundation of China (Grant no. 61973041) and the National Key Research and Development Program of China (Grant no. 2019YFB1705403).

\section{References}

[1] J. Yu, L. Gao, G. Yu, K. Liu, and Y. Guo, "Planetary gear fault identification based on SDAE and GRUNN," Vibration and Shock, vol. 40, no. 2, pp. 156-163, 2021.

[2] H. Gao and A. Fan, "Research on the fault of planetary gearbox based on CEEMD and CPSO-ELM," Automation Technology and Application, vol. 40, no. 1, pp. 95-100, 2021.

[3] Z. Wang, L. Yao, X. Qi, J. Zhang, and J. Zheng, "Fault diagnosis of planetary gearbox based on parameter optimization variational modal decomposition and multi-domain manifold learning," Vibration and Shock, vol. 40, no. 1, pp. 110-118, 2021.

[4] H. Li, Z. Qi, and J. Hu, "Research on intelligent judgment method of planetary gearbox tooth surface wear fault based on FFT-DBN," Measurement \& Control Technology, vol. 39, no. 12, pp. 50-54, 2020.

[5] Y. Li, Z. Jiang, H. Liang, and X. Xu, "Fault diagnosis of planetary gearbox based on EEMD symmetric differential energy spectrum," Modular Machine Tool and Automatic Manufacturing Technology, vol. 47, no. 10, pp. 122-125, 2020.

[6] Q. Zhang, H. Li, and S. Li, "Application of Time-Frequency Features and PSO-SVM in Fault Diagnosis of Planetary Gearbox," in Proceedings of the 2021, Springer Science and Business Media Deutschland GmbH, vol. 1274, Xi'an, China, September 2021.

[7] Z. Wang, H. Huang, and Y. Wang, "Fault diagnosis of planetary gearbox using multi-criteria feature selection and heterogeneous ensemble learning classification," Measurement: Journal of the International Measurement Confederation, vol. 173, p. 173, 2021.

[8] X. Zhang, L. Lin, S. Liu, and J. Lei, "Empirical wavelet transform based on energy peak location and its application in weak bearing fault diagnosis," Journal of Xi'an Jiaotong University, vol. 12, no. 8, pp. 1-9, 2021.
[9] X. Li, Z. Ma, D. Kang, and X. Li, "Fault diagnosis for rolling bearing based on VMD-FRFT," Measurement: Journal of the International Measurement Confederation, vol. 155, 2020.

[10] W. Liu, "Digital watermarking algorithm based on fractional discrete wavelet transform," Shandong Industrial Technology, vol. 4, no. 9, p. 164, 2019.

[11] S. K. Upadhyay and K. Khatterwani, "Fractional wavelet transform through heat equation," Journal of Thermal Stresses, vol. 42, no. 11, pp. 1386-1414, 2019.

[12] Y. Ding, Y. He, B. Li, and J. Cui, "Inverter fault diagnosis based on wavelet packet and quantum neural network," Journal of Chongqing University of Technology (Natural Science), vol. 35, no. 4, pp. 152-158, 2021.

[13] J. Xu, Research on Bearing Fault Diagnosis Method of Wind Turbine Based on Convolutional Neural Network, Zhejiang University, HangZhou, China, 2020.

[14] X. Xiong, J. Wang, Y. Zhang, Q. Guo, and S. Zong, “A twodimensional convolutional neural network optimization method for bearing fault diagnosis," Proceedings of the Chinese Society for Electrical Engineering, vol. 39, no. 15, pp. 45584568, 2019.

[15] C. Huang, Research on Optimal Method of Rolling Bearing Fault Diagnosis Combining Time-Frequency Analysis and Convolutional Neural Network, Zhejiang University, HangZhou, China, 2019. 